地球物理学

\title{
漂移理论解释 $F_{2}$ 层异常的可能性
}

\author{
李 緝 熙
}

(中国科学技术大学)

电离层状态与无线电通讯密切相关，因 此，电离层物理学就成为近代地球物理学发 展极为迅速的学科之一. 然而値得注意的 是，直到目前为止，电离层物理学工作者尚 无法解释电离层主要区域 $\left(\mathrm{F}_{2}\right.$ 层) 的运动特 性. 1931 年, Chapman 提出了电离层形成 理论．这个理论基本上可以解释 $\mathrm{E}$ 层和 $\mathrm{F}_{1}$ 层 的主要性质, 但与 $\mathrm{F}_{2}$ 层却有较大的偏离, 这就是所谓“ $F_{2}$ 层异常”. 为了揭示这一电离 层空间物理过程的实质，许多工作者作了很 多努力, 提出了各种假说和理论, 其中的绝 大部分都由于与观测事实不符而先后被否定 过. 1947-1948 年期间，Martyn 建立了漂 移理论 ${ }^{[1-4]}$. 这个理论曾得到一致的好评, 在几次重要的国际性电离层会议上都曾被加 以肯定，直到目前为止，沟有人提出过异 议。不少人溑循 Martyn 的思想, 作了某些 定量的计算, 其中, Fejer 的工作最为出色 ${ }^{[5]}$.

我们认为，漂移理论是无效的:

（1）在漂移理论中，有关漂移速度的定 义有原则性的错误.

电离层介质可以认为是一种特殊的等离 子体，因此，它的漂移速度应当是

$$
\begin{aligned}
\mathbf{v} & =\frac{1}{\rho_{+}+\rho_{e}}\left(\rho_{+} \mathbf{v}_{+}+\rho_{c} \mathbf{v}_{e}\right) \\
& \approx \mathbf{v}_{+}+\frac{m_{e}}{m_{+}} \mathbf{v}_{e}
\end{aligned}
$$

其中 $\mathbf{v}, \rho, m$ 分別表示漂移速度、密度和质 量, 而角码 “十” 和 “ $e$ ” 分別表示正离子和 电子的物理量. 假定电离层介质呈电中性. Martyn 片面地将正离子的运动速度当作等离 子体的漂移速度; Fejer 则将电子的运动速 度当作等离子体的漂移速度. 这都是不正确 的.

在外力 $\mathbf{F}$ 和地磁场 $\mathbf{H}_{0}$ 的作用下，第 $i$ 种带电粒子的运动方程为

$$
m_{i} \frac{d \mathbf{v}_{i}}{d t}+m_{i} \nu_{i} \mathbf{v}_{i}=\frac{e_{i}}{c} \mathbf{v}_{i} \times \mathbf{H}_{0}+\mathbf{F}_{i}
$$

其中 $e$ 为一个带电粒子的电荷量， $v$ 为碰撞 频率, $c$ 为光速. 如果所有漂移运动都是由 于潮汐力所引起的, 则上式中左方第一项比 第二项小得多，因而可以略去，则

$$
m_{i} \nu_{i} \mathbf{v}_{i}=\frac{e_{i}}{c} \mathbf{v}_{i} \times \mathbf{H}_{0}+\mathbf{F}_{i}
$$

设 $\mathbf{H}_{0}$ 为偶极子场，由 (3) 式可分別求出 $\mathbf{v}_{+}$ 和 $\mathbf{v}_{e}$ ，代入 (1) 式，则得到在球座标中的漂 移速度分量

$$
\begin{gathered}
{\left[\begin{array}{l}
v_{R} \\
v_{\theta} \\
v_{\lambda}
\end{array}\right]=\frac{1}{m_{+}} \sum_{+, e} \frac{1}{v_{i}\left(v_{i}^{2}+\omega_{i}^{2}\right)} \times} \\
\times\left[\begin{array}{ccc}
v_{i}^{2}+\omega_{R i}^{2} & \omega_{R i} \omega_{\theta i} & -\omega_{\theta i} \nu_{i} \\
\omega_{R i} \omega_{\theta i} & v_{i}^{2}+\omega_{\theta i}^{2} & \omega_{R i} \nu_{i} \\
\omega_{\theta_{i}} \nu_{i} & -\omega_{R i} \nu_{i} & \nu_{i}^{2}
\end{array}\right]\left[\begin{array}{l}
F_{R i} \\
F_{\theta_{i}} \\
F_{\lambda_{i}}
\end{array}\right]
\end{gathered}
$$


其中 $\omega$ 为磞旋频率.

（2）漂移运动在整个电离层状态中所起 的作用应当按照下列的制別式来加以确定.

电离层状态的变化遵守连续方程

$$
\frac{\partial N}{\partial t}=J-\beta N-\nabla \cdot(N \mathbf{v})
$$

其中 $N$ 为电子浓度, $J$ 为电离速率， $\beta$ 为附 着系数。在这里只考虑漂移运动速度 $\mathbf{v}$. 在 $F_{2}$ 层条件下，上式可化简为

$$
\frac{\partial N}{\partial t}=J-(\beta+\nabla \cdot \mathbf{v}) N
$$

所以, 当

$$
\beta \gg|\nabla \cdot \mathbf{v}|
$$

时, 漂移运动是不重要的, 漂移理论失效. 而当

$$
\beta \ll|\nabla \cdot \mathbf{v}|
$$

时, 漂移运动是重要的, 漂移理论可能有成 效.

Ratcliffe 等 ${ }^{[6]}$ 曾对 $\beta$ 值作过详细的论证， 在 $F_{2}$ 层

$$
\beta \sim 10^{-4} \text { 秒 }^{-1}
$$

下面我们来计算一下各种漂移运动所引 起的速度散度，就能够从(7)一(9)式来刵断 漂移运动有多大威力。

（3）极化电场引起 $F_{2}$ 层的漂移运动微 不足道. 让我们引用 Fejer 计算的在发电机 区的极化势

$$
\begin{gathered}
\psi=-R\left(\psi_{a} \sin 2 \lambda+\psi_{b} \cos 2 \lambda\right) \\
\lambda=\Omega t+\vec{\lambda}+77^{\circ}
\end{gathered}
$$

其中 $t$ 为世界时， $\Omega$ 为地球自转角速度， $\bar{\lambda}$ 为从格林威治向东算起的经度, $R$ 为地心距 离, $\psi_{a}$ 和 $\psi_{b}$ 是磁纬的函数. 漂移理论认为, 这个极化场将通过高导电率的磁力线传递到 $\mathrm{F}_{2}$ 层, 从而引起 $\mathrm{F}_{2}$ 层电离体的漂移. 按照 这一想法，经过一定运算并采用合理的电离 层模式进行适当的化简后，从(4)式得到

$$
\begin{aligned}
{\left[\begin{array}{l}
v_{R} \\
v_{\theta} \\
v_{k}
\end{array}\right]_{E}=\frac{|e|}{m_{+} \nu} } & {\left[\begin{array}{ccc}
\frac{\omega_{\theta e}^{2}}{\omega_{e}^{2}} & -\frac{\omega_{R e} \omega_{\theta_{e}}}{\omega_{e}^{2}} & 0 \\
-\frac{\omega_{R e} \omega_{\theta_{e}}}{\omega_{e}^{2}} & \frac{\omega_{R e}^{2}}{\omega_{e}^{2}} & 0 \\
0 & 0 & 1
\end{array}\right] \times } \\
& \times\left[\begin{array}{c}
E_{R} \\
E_{\theta} \\
E_{\lambda}
\end{array}\right]
\end{aligned}
$$

注意到 $\mathbf{E}=-\nabla \psi$, 经过单调而又很繁圥的 运算, 从(10)、(11)式得

$$
(\nabla \cdot \mathbf{v})_{E}=A(\theta) \sin 2 \lambda+B(\theta) \cos 2 \lambda \quad(12)
$$

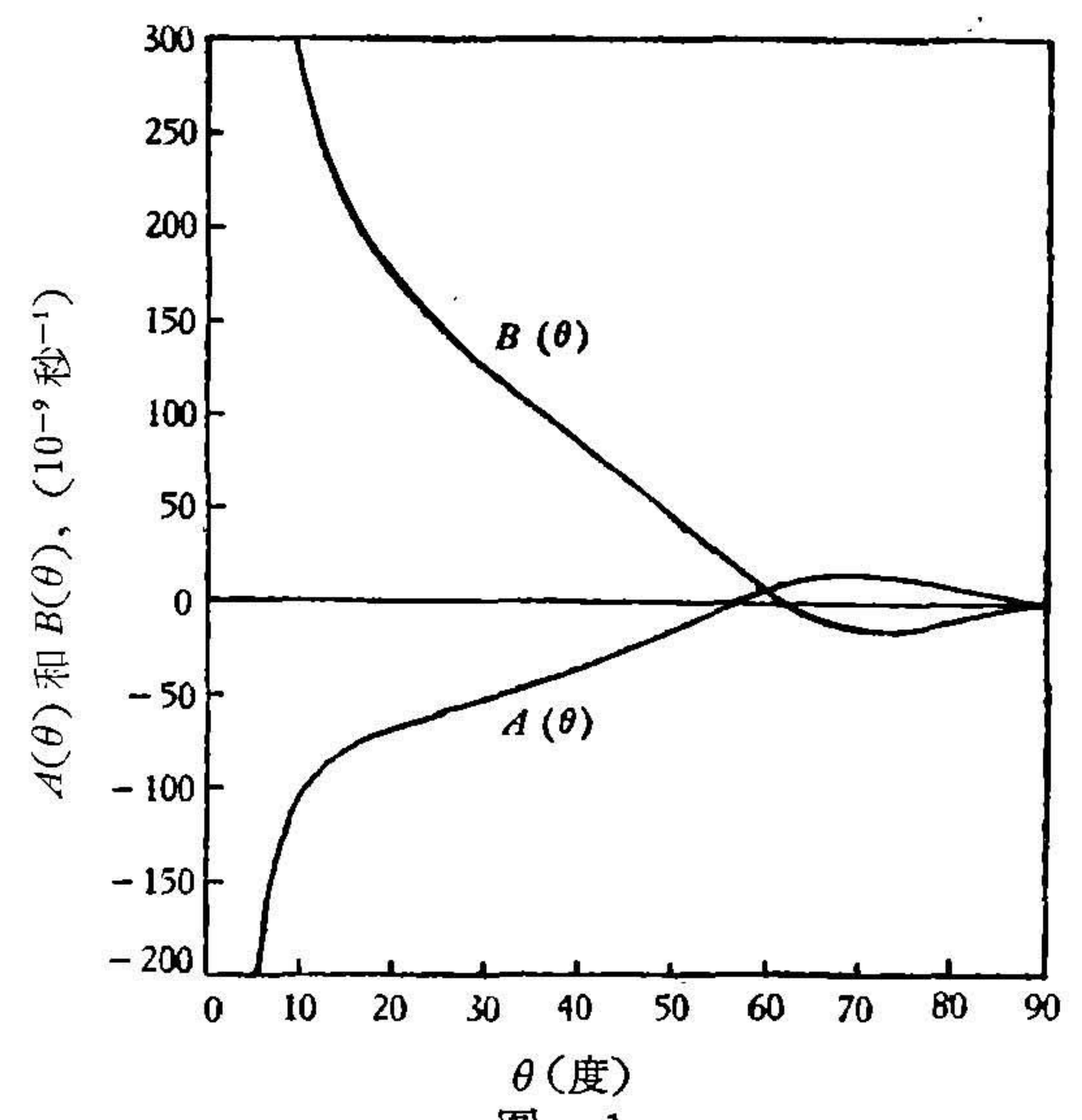

图 1

图 1 画出 $A(\theta)$ 和 $B(\theta)$ 曲线, 可以看 出, 在同一子午圈上, 在磁纬 $30^{\circ}$ 左右, $(\nabla \cdot \mathbf{v})_{E}$ 改变了符号. 这一点与发电机区的 特性相倣。

最重要的结果是在磁纬低于 $60^{\circ}$ 左右的 大部分区域， $(\nabla \cdot \mathbf{v})_{E} \sim 10^{-9}-10^{-8}$ 秒 ${ }^{-1}$. 显 然它完全满足 (7) 式的条件, 因而漂移运动 不重要. 只有在极冠很小一部分, $(\nabla \cdot \mathbf{v})_{E}$ 才是可观的，（8）式才能成立, 亦師漂移运 动才是重要的.

（4）潮汐风场本身引起的漂移运动也是 微不足道的. 此时风力和风速有如下关系: 


$$
\mathbf{F}_{i}=m_{i} \nu_{i} \nabla_{W}
$$

其中 $\mathbf{v}_{W}$ 为风速. 选择合理的电离层模式, 不难从(4)、(13)式得到

$$
\left[\begin{array}{l}
v_{R} \\
v_{\theta} \\
v_{2}
\end{array}\right]_{W} \approx\left[\begin{array}{l}
v_{W R} \\
v_{W \theta} \\
v_{W R}
\end{array}\right]
$$

也就是说，在潮汐风场的作用下，带电粓子 完全与中性粒子一样运动，沟有什么特殊 性. 这是由于，对于正离子来说，磁旋频率 比碰撞频率小得多，正离子受磁场的控制作 用可以略去不计；对于电子来说，虽然磁旋 频率大于碰撞频率，但由于电子的质量小， 它在整个等离子体漂移运动中的贡献不大， 因而对潮汐风场来说，也表现不出来电离层 介质的各向异性。

（14）式的结果与 Martyn 大不相同。事 实上，只要将

$$
\omega_{+} \ll \nu
$$

的条件代入 $\operatorname{Martyn}^{[1]}$ 的 (1)-(3)式中去, 就 得到我们的 (14) 式. 在 $F_{2}$ 层, (15) 式是充 分成立的.

对 (14) 式取散度，利用著名的 Simpson 潮汐风场的公式，则不难算出

$$
(\nabla \cdot \mathbf{v})_{W}=C(\theta) \cos 2 \lambda
$$

图 2 是 $C(\theta)$ 曲线. 可以看出, $C(\theta)$ 的 量级大致为 $10^{-6}$ 秒 $^{-1}$. 因此，（7）式完全成 立, 漂移运动并不重要。

（5）按照 Fejer 的意见， $F_{2}$ 层的潮汐放 大倍数约为 60 , 在以上所有计算中, 我们 已假定为 100 . 尽管目前人们对潮汐放大倍 数还搞不清楚, 我们的结论还是可信的. 因 为, 如果希望借助于这一点来维持住漂移理 论, 那么, 对于极化电场引起的漂移来说, 潮汐振幅的放大倍数至少需达 $10^{6}$ 倍; 对于 潮汐风场引起的漂移来说, 至少需达 $10^{4}$ 倍,
这大概是不可能的.

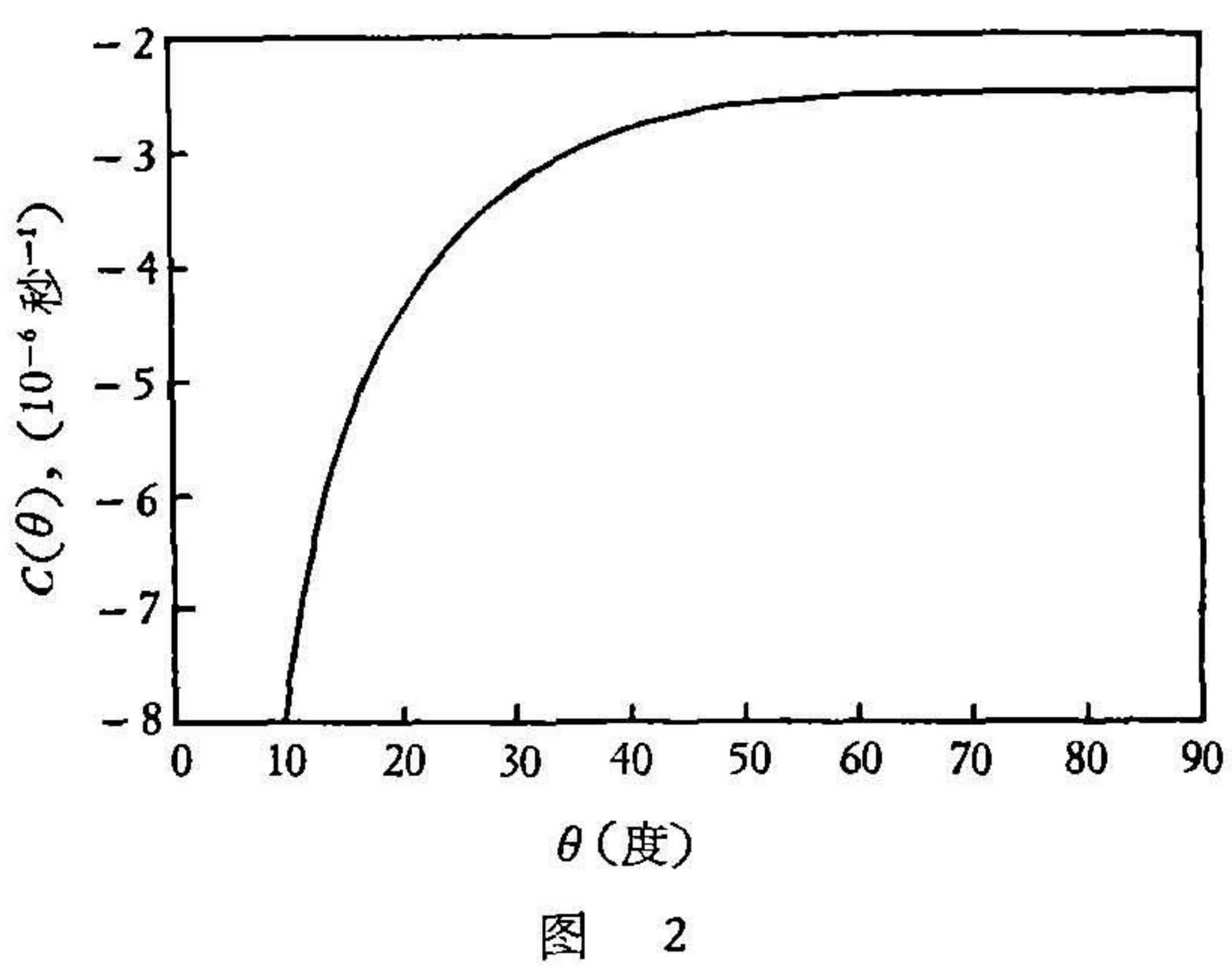

在 $\mathrm{F}_{2}$ 层，除了可能有上述两种漂移之 外，还可能有发电机场引起的漂移，本文不 再继续对它进行讨论. 可以想象，它对漂移 速度散度的贡献一定不会超过极化电场的贡 献，因为前者的纬度分布比后者平滑的多 (并且有同一数量级).

总之, 在 $\mathrm{F}_{2}$ 层, 漂移运动是微不足道 的. 漂移理论是无效的. 事实上, Martyn ${ }^{[1]}$ 的漂移速度表达式中就含有未知的潮汐放大 倍数, 可是, 这一个原则性的问题在过去十 多年中一直不曾引起人们的重视, 相反地, 却由于漂移理论表面上能解释部分事实而迷 信它.

必须指出， $F_{2}$ 层异常可能是由许多物理 机制引起的综合过程, 企图用单一的物理机 制来包罗万象地解释它是不可能的.

\section{参考文 献}

[1] Martyn, D. F., Proc. Roy. Soc., A189, 241 (1947).

[2] —, Ibid, A190, 273 (1947).

[3] —, Ibid, A194, 429 (1948).

[4] - Ibid, A194, 445 (1948).

[5] Fejer, J. A., J. Atmos. Terr. Phys., 4, 184 (1953).

[6] Ratcliffe, J. A. et al., Phil. Trans. Roy. Soc., A248, 621 (1956). 